\title{
ENUMERATION OF COMMUTING PAIRS IN LIE ALGEBRAS OVER FINITE FIELDS
}

\author{
JASON FULMAN AND ROBERT GURALNICK
}

\begin{abstract}
Feit and Fine derived a generating function for the number of ordered pairs of commuting $n \times n$ matrices over the finite field $\mathbb{F}_{q}$. This has been reproved and studied by Bryan and Morrison from the viewpoint of motivic Donaldson-Thomas theory. In this note we give a new proof of the Feit-Fine result, and generalize it to the Lie algebra of finite unitary groups and to the Lie algebra of odd characteristic finite symplectic groups. We extract some asymptotic information from these generating functions. Finally, we derive generating functions for the number of commuting nilpotent elements for the Lie algebras of the finite general linear and unitary groups, and of odd characteristic symplectic groups.
\end{abstract}

\section{INTRODUCTION}

Let $G_{0}=1$, and for $n \geq 1$, let $G_{n}$ be the number of ordered pairs of commuting $n \times n$ matrices (not necessarily invertible) over $\mathbb{F}_{q}$. Feit and Fine $[\mathrm{FF}]$ proved the following theorem.

\section{Theorem 1.1.}

$$
\sum_{n \geq 0} \frac{G_{n} u^{n}}{|\operatorname{GL}(n, q)|}=\prod_{i \geq 1} \prod_{l \geq 0} \frac{1}{1-u^{i} / q^{l-1}} .
$$

Theorem 1.1 was reproved and refined by Bryan and Morrison [BM] from the viewpoint of motivic Donaldson-Thomas theory, using "power structures". See also BBS].

In this paper we give a new proof of the Feit-Fine theorem. Answering a question of Matt Young (an expert in Donaldson-Thomas theory), we obtain analogs of the Feit-Fine theorem for unitary groups and for odd characteristic symplectic groups. More precisely, let $U_{0}=1$ and for $n \geq 1$, let $U_{n}$ be the number of ordered pairs of commuting elements of the Lie algebra of $\mathrm{GU}(n, q)$. We will show that

$$
\sum_{n \geq 0} \frac{U_{n} u^{n}}{|\mathrm{GU}(n, q)|}=\prod_{i \geq 1} \prod_{l \geq 0} \frac{1}{1-(-1)^{l} u^{i} / q^{l-1}}
$$

Let $S_{0}=1$ and for $n \geq 1$, let $S_{n}$ be the number of ordered pairs of commuting elements of the Lie algebra of $\operatorname{Sp}(2 n, q)$ in odd characteristic. We will prove

Date: October 17, 2016. 
that

$$
\sum_{n \geq 0} \frac{S_{n} u^{n}}{|\operatorname{Sp}(2 n, q)|}=\frac{\prod_{i \geq 1}\left(1+u^{i}\right)}{\prod_{i \geq 1} \prod_{l \geq 0}\left(1-u^{i} / q^{2 l-1}\right)} .
$$

Our methods will also work for odd characteristic orthogonal groups, but we do not treat them as this is quite tedious and the ideas are the same as for odd characteristic symplectic groups. On the other hand, for various reasons (e.g. nilpotent classes are no longer determined by their Jordan form even in the algebraic group and the description of semisimple classes is different as well), even characteristic symplectic and orthogonal groups are much trickier, and are beyond the scope of this paper.

It is easy to extract asymptotic information from our generating functions. For example from the original Feit-Fine result, we will conclude that for $q$ fixed and $n$ tending to infinity,

$$
\lim _{n \rightarrow \infty} \frac{G_{n}}{q^{n^{2}+n}}=\prod_{j \geq 1}\left(1-1 / q^{j}\right)^{-j} .
$$

This is consistent with the fact that the variety of $n \times n$ commuting matrices over an algebraically closed field has dimension $n^{2}+n$.

Finally, we derive an analog of the Feit-Fine theorem for nilpotent commuting pairs. Let $N G_{0}=1$, and for $n \geq 1$, let $N G_{n}$ be the number of ordered pairs of commuting nilpotent $n \times n$ matrices over $\mathbb{F}_{q}$. We will prove that

$$
\sum_{n \geq 0} \frac{N G_{n} u^{n}}{|\operatorname{GL}(n, q)|}=\prod_{i \geq 1} \prod_{l \geq 1} \frac{1}{1-u^{i} / q^{l}} .
$$

We also obtain generating functions for $N U_{n}$ (the number of ordered pairs of commuting nilpotent elements of the Lie algebra of $\mathrm{GU}(n, q)$ ), and for $N S_{n}$ (the number of ordered pairs of commuting nilpotent elements of the Lie algebra of the odd characteristic symplectic group $\operatorname{Sp}(2 n, q)$ ).

We note there has been quite a lot of work on commuting varieties. One of the first results in this area is the result of Motzin and Taussky MT] that the variety of commuting pairs in $\operatorname{Mat}(n, k)$ with $k$ algebraically closed is irreducible of dimension $n^{2}+n$. This was extended by Richardson R2 to reductive Lie algebras $\mathfrak{g}$ in characteristic 0 and by Levy [Le] in good characteristic with an extra mild assumption. It follows easily that in this case the dimension of the commuting variety is $\operatorname{dim} \mathfrak{g}+$ rank $\mathfrak{g}$. Recall that good characteristic means any characteristic in type $A$, characteristic not 2 for groups of type $B, C, D$ and characteristic not 2 or 3 for $G_{2}, F_{4}, E_{6}$ and $E_{7}$ and characteristic not 2,3 or 5 for $E_{8}$. Very good characteristic means good characteristic for groups not of type $A$ and characteristic not dividing $n+1$ for groups of type $A_{n}$.

There has also been quite a bit of study for the variety of commuting nilpotent pairs in a simple Lie algebra $\mathfrak{g}$ corresponding to a Dynkin diagram in good characteristic. The components are in bijection with the set of distinguished orbits of nilpotent elements (i.e. the centralizer in the group 
contains no nontrivial torus) and all components have dimension dim $\mathfrak{g}$ (under the assumption that the characteristic is very good). This was proved by Premet $[\mathrm{Pr}]$ after some earlier results of Baranovosky [Ba] and Basili [Bs] for $\mathfrak{g l}_{n}$ (with some restrictions on the characteristic). In particular, this variety is irreducible for $\operatorname{Mat}(n, k)$. It will have a large number of components for some of the other simple Lie algebras. For example when $\mathfrak{g}=\mathfrak{s p}(2 n, k)$ and the characteristic of $k$ is not 2, the number of irreducible components is the number of partitions of $n$ with all parts of distinct sizes.

Our methods would give similar answers for the special orthogonal Lie algebras in characteristic not 2 . We do not work out the details of this case and leave it to the interested reader.

The organization of this paper is as follows. Section 2 treats the general linear groups. It gives a new proof of the Feit-Fine theorem, obtains asymptotic information from it, and enumerates commuting pairs of nilpotent matrices. Section 3 studies the Lie algebra of the finite unitary groups $\mathrm{GU}(n, q)$, giving unitary analogs of all the results in Section 2, Section 4 studies the Lie algebra of the finite symplectic groups $\operatorname{Sp}(2 n, q)$ in the case of odd characteristic, giving symplectic analogs of all the results in Section 2,

\section{General Linear Groups}

We use the following notation about partitions. Let $\lambda$ be a partition of some non-negative integer $|\lambda|$ into parts $\lambda_{1} \geq \lambda_{2} \geq \cdots$. Let $m_{i}(\lambda)$ be the number of parts of $\lambda$ of size $i$, and let $\lambda^{\prime}$ be the partition dual to $\lambda$ in the sense that $\lambda_{i}^{\prime}=m_{i}(\lambda)+m_{i+1}(\lambda)+\cdots$. It is also useful to define the diagram associated to $\lambda$ by placing $\lambda_{i}$ boxes in the $i$ th row. We use the convention that the row index $i$ increases as one goes downward. So the diagram of the partition (5441) is

and $\lambda_{i}^{\prime}$ can be interpreted as the size of the $i$ th column.

The notation $(1 / q)_{r}$ will denote $(1-1 / q)\left(1-1 / q^{2}\right) \cdots\left(1-1 / q^{r}\right)$.

The next two lemmas are well known and will be useful. Lemma 2.1 is due to Euler and is proved on page 19 of $[\mathrm{A}$.

\section{Lemma 2.1.}

$$
\sum_{m \geq 0} \frac{u^{m}}{(1 / q)_{m}}=\prod_{l \geq 0} \frac{1}{1-u / q^{l}} .
$$

Lemma 2.2 is elementary. In its statement, and for the rest of the paper, we let $d(\phi)$ denote the degree of a polynomial $\phi$. For completeness, we include a proof. 


\section{Lemma 2.2.}

$$
\prod_{\phi}\left(1-u^{d(\phi)}\right)=1-u q,
$$

where the product is over all monic, irreducible polynomials over the finite field $\mathbb{F}_{q}$.

Proof. By unique factorization in $\mathbb{F}_{q}[x]$, the coefficient of $u^{n}$ in the reciprocal of the left hand side is the number of degree $n$ monic polynomials with coefficients in $\mathbb{F}_{q}$. This is equal to $q^{n}$, which is the coefficient of $u^{n}$ in $(1-u q)^{-1}$.

We let $\operatorname{Mat}(n, q)$ denote the set of $n \times n$ matrices (not necessarily invertible) over the field $\mathbb{F}_{q}$. Recall the rational canonical form of an element of $\operatorname{Mat}(n, q)$. This associates to each monic, irreducible polynomial $\phi$ over $\mathbb{F}_{q}$ a partition $\lambda_{\phi}$ such that

$$
\sum_{\phi} d(\phi)\left|\lambda_{\phi}\right|=n
$$

For further background on rational canonical forms, one can consult Chapter 6 of $[\mathrm{H}]$.

Lemma 2.3 is proved in Stong [S], and calculates the number of elements of $\operatorname{Mat}(n, q)$ with given rational canonical form.

Lemma 2.3. Suppose that

$$
\sum_{\phi} d(\phi)\left|\lambda_{\phi}\right|=n
$$

so that $\left\{\lambda_{\phi}\right\}$ is a possible rational canonical form of an element of $\operatorname{Mat}(n, q)$. Then the number of elements of $\operatorname{Mat}(n, q)$ with rational canonical form $\left\{\lambda_{\phi}\right\}$ is equal to

$$
\frac{|\operatorname{GL}(n, q)|}{\prod_{\phi} q^{d(\phi) \sum_{i}\left(\lambda_{\phi, i}^{\prime}\right)^{2}} \prod_{i}\left(1 / q^{d(\phi)}\right)_{m_{i}\left(\lambda_{\phi}\right)}} .
$$

(Recall that $\lambda_{\phi, i}^{\prime}$ is the size of the ith column of the partition $\lambda_{\phi}$ ).

Lemma 2.4 counts the number of elements of $\operatorname{Mat}(n, q)$ which commute with a given element of $\operatorname{Mat}(n, q)$ which has rational canonical form $\left\{\lambda_{\phi}\right\}$.

Lemma 2.4. Let $x$ be an element of $\operatorname{Mat}(n, q)$ with rational canonical form $\left\{\lambda_{\phi}\right\}$. Then the number of elements of $\operatorname{Mat}(n, q)$ which commute with $x$ is equal to

$$
\prod_{\phi} q^{d(\phi) \sum_{i}\left(\lambda_{\phi, i}^{\prime}\right)^{2}}
$$

Proof. Note that the exponent in the statement of the result is precisely the dimension of the centralizer of $x$ (either in $\operatorname{Mat}(n, q)$ or $\operatorname{Mat}(n, k)$ where $k$ is the algebraic closure of our finite field). Note that the centralizer breaks up into the product of the centralizers corresponding to each irreducible 
polynomial $\phi$. By passing to a splitting field for $\phi$, it suffices to assume that $\phi$ has degree 1 and indeed that $\phi=x$.

Now we can decompose the underlying space $V$ into a direct sum $V_{1} \oplus$ $\ldots \oplus V_{m}$ where $x$ acts via a single Jordan block on $V_{i}$ for size $\lambda_{i}$. Then the dimension of the centralizer is the sum of the dimensions of of $\operatorname{Hom}_{k[x]}\left(V_{i}, V_{j}\right)$. Since $V_{j}$ and $V_{j}$ are cyclic $k[x]$-modules, this dimension is the minimum of $\lambda_{i}, \lambda_{j}$. Clearly

$$
\sum_{i, j \geq 1} \min \left(\lambda_{i}, \lambda_{j}\right)=2 \sum_{k<l} k m_{k}(\lambda) m_{l}(\lambda)+\sum_{i} i m_{i}(\lambda)^{2} .
$$

This is equal to $\sum_{i}\left(\lambda_{i}^{\prime}\right)^{2}$, as can be seen by writing

$$
\lambda_{i}^{\prime}=m_{i}(\lambda)+m_{i+1}(\lambda)+\cdots .
$$

Now, we reprove the Feit-Fine theorem.

Proof. We let $\left[u^{n}\right] f(u)$ denote the coefficient of $u^{n}$ in an expression $f(u)$. It follows from Lemmas 2.3 and 2.4 that $G_{n}$ (the number of ordered pairs of commuting elements of $\operatorname{Mat}(n, q))$ is equal to

$$
|\mathrm{GL}(n, q)|\left[u^{n}\right] \prod_{\phi} \sum_{\lambda} \frac{u^{|\lambda| d(\phi)}}{\prod_{i}\left(1 / q^{d(\phi)}\right)_{m_{i}(\lambda)}},
$$

where the product is over all monic irreducible polynomials $\phi$ over $\mathbb{F}_{q}$. This is equal to

$$
|\mathrm{GL}(n, q)|\left[u^{n}\right] \prod_{\phi} \prod_{i \geq 1} \sum_{m \geq 0} \frac{u^{i m \cdot d(\phi)}}{\left(1 / q^{d(\phi)}\right)_{m}} .
$$

By Lemma 2.1, this is equal to

$$
|\mathrm{GL}(n, q)|\left[u^{n}\right] \prod_{\phi} \prod_{i \geq 1} \prod_{l \geq 0} \frac{1}{1-u^{i \cdot d(\phi)} / q^{l \cdot d(\phi)}} .
$$

Switching the order of the products and applying Lemma 2.2 shows that this is equal to

$$
\begin{aligned}
& |\mathrm{GL}(n, q)|\left[u^{n}\right] \prod_{i \geq 1} \prod_{l \geq 0} \prod_{\phi} \frac{1}{1-u^{i \cdot d(\phi)} / q^{l \cdot d(\phi)}} \\
= & |\operatorname{GL}(n, q)|\left[u^{n}\right] \prod_{i \geq 1} \prod_{l \geq 0} \frac{1}{1-u^{i} / q^{l-1}} .
\end{aligned}
$$

This completes the proof.

Next we derive asymptotic information from the Feit-Fine generating function.

Given a power series $f(u)$, let $\left[u^{n}\right] f(u)$ denote the coefficient of $u^{n}$ in $f(u)$. The following lemma will be useful. 
Lemma 2.5. If the Taylor series of $f$ around 0 converges at $u=1$, then

$$
\lim _{n \rightarrow \infty}\left[u^{n}\right] \frac{f(u)}{1-u}=f(1)
$$

Proof. Write the Taylor expansion $f(u)=\sum_{n=0}^{\infty} a_{n} u^{n}$. Then observe that $\left[u^{n}\right] \frac{f(u)}{1-u}=\sum_{i=0}^{n} a_{i}$.

Theorem 2.6. For $q$ fixed,

$$
\lim _{n \rightarrow \infty} \frac{G_{n}}{q^{n^{2}+n}}=\prod_{j \geq 1}\left(1-1 / q^{j}\right)^{-j}
$$

Proof. We know that

$$
\begin{aligned}
G_{n} & =|\operatorname{GL}(n, q)|\left[u^{n}\right] \prod_{i \geq 1} \prod_{l \geq 0} \frac{1}{1-u^{i} / q^{l-1}} \\
& =|\operatorname{GL}(n, q)| q^{n}\left[u^{n}\right] \prod_{i \geq 1} \prod_{l \geq 0} \frac{1}{1-u^{i} / q^{i+l-1}} \\
& =q^{n^{2}+n}(1 / q)_{n}\left[u^{n}\right] \prod_{i \geq 1} \prod_{l \geq 0} \frac{1}{1-u^{i} / q^{i+l-1}} .
\end{aligned}
$$

Thus

$$
\lim _{n \rightarrow \infty} \frac{G_{n}}{q^{n^{2}+n}}=\prod_{j \geq 1}\left(1-1 / q^{j}\right) \lim _{n \rightarrow \infty}\left[u^{n}\right] \prod_{i \geq 1} \prod_{l \geq 0} \frac{1}{1-u^{i} / q^{i+l-1}} .
$$

Since the $i=1, l=0$ case of $1 /\left(1-u^{i} / q^{i+l-1}\right)$ is equal to $1 /(1-u)$, it follows from Lemma 2.5 that

$$
\lim _{n \rightarrow \infty}\left[u^{n}\right] \prod_{i \geq 1} \prod_{l \geq 0} \frac{1}{1-u^{i} / q^{i+l-1}}=\prod_{j \geq 1}\left(1-1 / q^{j}\right)^{-j-1},
$$

which implies the theorem.

Remark: Let $\operatorname{Com}(G)$ denote the number of ordered pairs of commuting elements of a finite group $G$. It is easy to see that $\operatorname{Com}(G)$ is equal to $k(G)|G|$, where $k(G)$ is the number of conjugacy classes of $G$. From [FG], $\lim _{n \rightarrow \infty} \frac{k(\mathrm{GL}(n, q))}{q^{n}}=1$. Thus,

$$
\lim _{n \rightarrow \infty} \frac{\operatorname{Com}(\operatorname{GL}(n, q))}{q^{n^{2}+n}}=\lim _{n \rightarrow \infty} \frac{|\operatorname{GL}(n, q)|}{q^{n^{2}}}=\prod_{j \geq 1}\left(1-1 / q^{j}\right) .
$$

To close this section, we enumerate pairs of commuting nilpotent $n \times n$ matrices. Let $N G_{0}=1$, and for $n \geq 1$, let $N G_{n}$ be the number of ordered pairs of commuting $n \times n$ nilpotent matrices over $\mathbb{F}_{q}$.

Theorem 2.7.

$$
\sum_{n \geq 0} \frac{N G_{n} u^{n}}{|\operatorname{GL}(n, q)|}=\prod_{i \geq 1} \prod_{l \geq 1} \frac{1}{1-u^{i} / q^{l}}
$$


The next result gives the number of nilpotent elements commuting with a given nilpotent element.

Lemma 2.8. The number of nilpotent $n \times n$ matrices that commute with a fixed nilpotent matrix of type $\lambda$ is equal to

$$
q^{\sum_{i}\left(\lambda_{i}^{\prime}\right)^{2}-\sum_{i} m_{i}(\lambda)}
$$

Proof. It is well known and easy that the centralizer in $\operatorname{Mat}(n, q)$ is

$$
\operatorname{Mat}\left(m_{1}, q\right) \times \ldots \times \operatorname{Mat}\left(m_{r}, q\right),
$$

modulo its nilpotent radical. From [Lh], the number of nilpotent matrices in $\operatorname{Mat}(m, q)$ is $q^{m^{2}-m}$. The result follows.

Now we prove Theorem 2.7 .

Proof. (Of Theorem 2.7) Lemma 2.3 gives that the number of $n \times n$ nilpotent matrices of Jordan type $\lambda$ is equal to

$$
\frac{|\operatorname{GL}(n, q)|}{q^{\sum_{i}\left(\lambda_{i}^{\prime}\right)^{2}} \prod_{i}(1 / q)_{m_{i}(\lambda)}} .
$$

Combining this with Lemma 2.8 gives that

$$
N G_{n}=|\operatorname{GL}(n, q)| \sum_{|\lambda|=n} \frac{1}{\prod_{i}(1 / q)_{m_{i}(\lambda)} q^{m_{i}(\lambda)}} .
$$

Thus

$$
N G_{n}=|\operatorname{GL}(n, q)|\left[u^{n}\right] \prod_{i \geq 1} \sum_{m \geq 0} \frac{u^{i m}}{(1 / q)_{m} q^{m}} .
$$

Now replacing $u$ by $u^{i} / q$ in Lemma 2.1 gives that

$$
\sum_{m \geq 0} \frac{u^{i m}}{(1 / q)_{m} q^{m}}=\prod_{l \geq 1} \frac{1}{1-u^{i} / q^{l}}
$$

It follows that

$$
N G_{n}=|\operatorname{GL}(n, q)|\left[u^{n}\right] \prod_{i \geq 1} \prod_{l \geq 1} \frac{1}{1-u^{i} / q^{l}},
$$

proving the theorem.

\section{UNITARY GROUPS}

We first prove some analogs of the GL case.

Let $F=\mathbb{F}_{q^{2}}$ and let $y^{q}=\bar{y}$ denote the $q$-Frobenius map on $F$. Let $F_{0}=\mathbb{F}_{q}$. Let $k$ denote the algebraic closure of $F$. We extend this map to an $F_{0}$ automorphism of $\operatorname{Mat}\left(n, q^{2}\right)$. Let $A^{\top}$ denote the transpose of $A$.

Since $\operatorname{GU}(n, q)$ are those matrices $U$ (in $\operatorname{Mat}\left(n, q^{2}\right)$ ) satisfying $U \bar{U}^{\top}=I$, the Lie algebra of $\operatorname{GU}(n, q)$ are those matrices $A \in \operatorname{Mat}\left(n, q^{2}\right)$ such that $\bar{A}^{\top}=-A$. We call these matrices skew Hermitian. We will occasionally write $\operatorname{GL}^{\epsilon}(n, q)$ for $\operatorname{GL}(n, q)$ with $\epsilon=+$ and $\operatorname{GU}(n, q)$ with $\epsilon=-$. 
Choose $\theta \in F$ so that $\theta \bar{\theta}=-1$ (if $F$ has characteristic 2, then take $\theta=1$ and if $F$ has odd characteristic, just take $\theta$ so that $\theta^{2} \in F_{0}$ is a nonsquare). Then $A$ skew Hermitian implies that $B:=\theta A$ is Hermitian (i.e. $\bar{B}^{\top}=B$ ). Let $\mathfrak{h}$ denote the set of Hermitian matrices. Note that the orbits of $\operatorname{GU}(n, q)$ on $\mathfrak{h}$ are in bijection with those of $\theta \mathfrak{h}$ and of course the set of commuting pairs are in bijection as well. Thus, we will obtain counts for the set of commuting pairs in $\mathfrak{h}$ (note that $\mathfrak{h}$ is a Jordan algebra).

We first observe that the orbits of $\mathrm{GU}(n, q)$ on $\mathfrak{h}$ are in bijection (which is not size preserving) with those of $\operatorname{GL}(n, q)$ on $\operatorname{Mat}(n, q)$.

Lemma 3.1. Let $A \in \operatorname{Mat}\left(n, q^{2}\right)$. Then $A$ is similar to an element of $\mathfrak{h}$ if and only if $A$ is similar to an element of $\operatorname{Mat}(n, q)$. Moreover, $A, B \in \mathfrak{h}$ are conjugate in $\mathrm{GL}(n, k)$ if and only if they are conjugate by an element of $\mathrm{GU}(n, q)$.

Proof. First suppose that $A \in \mathfrak{h}$. Since $\bar{A}^{\top}=A$ and $A$ and $A^{\top}$ are similar, it follows that $A$ and $\bar{A}$ are similar. Thus all invariant factors of $A$ are defined over $F_{0}$, whence $A$ is similar to an element of $\operatorname{Mat}(n, q)$.

Conversely, assume that $A \in \operatorname{Mat}(n, q)$. Since all nondegenerate Hermitian forms of dimension $n$ over $F$ are equivalent, it suffices to show that $A$ is self adjoint with respect to some Hermitian form - i.e. $A H=H \bar{A}^{\top}$ for some invertible Hermitian matrix $H$. By [TZ], $A S=S A^{\top}$ for some symmetric invertible matrix $S \in \operatorname{GL}(n, q)$. Of course, $S$ is Hermitian and $A$ is self adjoint with respect to the inner product determined by $S$, whence the first statement holds.

We now prove the last statement. Of course, if $A, B$ are conjugate via an element of $\mathrm{GU}(n, q)$ they are conjugate in $\operatorname{GL}(n, k)$. The other direction follows by the Lang-Steinberg theorem. If $B=U A U^{-1}$, then $A, B \in \mathfrak{h}$ implies that $U A U^{-1}=\bar{U}^{-\top} A \bar{U}^{\top}$. Thus, $\bar{U}^{\top} U$ centralizes $A$. By the LangSteinberg theorem (since the centralizer of $A$ in $\operatorname{GL}(n, k)$ is connected), $\bar{U}^{\top} U=\bar{X}^{\top} X$ for some $X$ centralizing $A$. Thus, $U X^{-1} A X U^{-1}=B$ and $U X^{-1} \in \mathrm{GU}(n, q)$.

Lemma 3.2. Let $A \in \mathfrak{h}$. Then the dimension of the centralizer (over $F_{0}$ ) of $A$ in $\mathfrak{h}$ is the same as the dimension of the centralizer (over $F$ ) of $A$ in $\operatorname{Mat}\left(n, q^{2}\right)$ (which is the same as the dimension of the centralizer in $\operatorname{Mat}(n, k))$.

Proof. We can write $\operatorname{Mat}\left(n, q^{2}\right)=\mathfrak{h} \oplus \alpha \mathfrak{h}$ (as $F_{0}$ spaces) for any element $\alpha \in F \backslash F_{0}$. Note that the map $X \rightarrow[A, X]$ preserves this decomposition. Thus, $C$ the centralizer of $A$ is a direct sum of its intersections with $\mathfrak{h}$ and $\alpha \mathfrak{h}$. Clearly, $\operatorname{dim}_{F_{0}} C \cap \mathfrak{h}=\operatorname{dim}_{F_{0}} \alpha \mathfrak{h}$. Letting $k C$ denote the centralizer of $A$ in $\operatorname{Mat}(n, k)$, we thus obtain $\operatorname{dim}_{F_{0}} C \cap \mathfrak{h}=\operatorname{dim}_{F} C=\operatorname{dim}_{k} k C$.

Lemma 3.3. Let $A \in \mathfrak{h}$. Let $f(x) \in F_{0}[x]$ denote the characteristic polynomial of $A$ and factor $f(x)=\prod_{i} \phi_{i}(x)^{e_{i}}$ where the $\phi_{i}$ are distinct monic 
irreducible polynomials in $F_{0}[x]$. Let $V$ denote the column space of dimension $n$ over $F$. Then $V$ is an orthogonal direct sum of spaces $V_{i}$ such that the characteristic polynomial of $A$ on $V_{i}$ is a power of $\phi_{i}$ with $\phi_{i} \neq \phi_{j}$.

Proof. By the previous lemma, for any given similarity class, there is a representative that is of the form given. Also by the previous lemma, the similarity class determines the orbit under the unitary group and the result follows.

We now want to determine the centralizer of $A \in \mathfrak{h}$. By the previous lemma, it suffices to do this under the assumption that the characteristic polynomial of $A$ is $\phi(x)^{m}$ where $\phi(x) \in F_{0}[x]$ is irreducible of degree $d$. The similarity class of such an $A$ (given $\phi(x)$ ) is determined by a partition of $m$. So write $m=\sum_{i} e_{i} \lambda_{i}$ where $\lambda_{1}>\lambda_{2}>\ldots$ and the partition has $e_{i}$ parts of length $\lambda_{i}$.

Lemma 3.4. Let $A \in \mathfrak{h}$. Let $G=\mathrm{GU}(n, q)$. Then $C_{G}(A)$ has unipotent radical $Q$ of order $q^{\ell}$ where $\ell$ is the dimension of the unipotent radical of $C_{\mathrm{GL}(n, k)}(A)$ and

$$
C_{G}(A) / Q \cong \prod_{i} \mathrm{GL}^{\epsilon}\left(e_{i}, q^{d}\right)
$$

where $\epsilon=+$ if $d$ is even and - if $d$ is odd.

Proof. Let $X$ be the centralizer of $A$ in $\operatorname{GL}(n, k)$. This has a unipotent radical $U$ and $Q$ is just the fixed points of $U$ under the endomorphism of $\mathrm{GL}(n, k)$ defining $G L^{\epsilon}(n, q)$. For any Steinberg-Lang endomorphism on a connected unipotent group, the size of the fixed point group is as given.

So we now focus on the reductive part of the centralizer. Let $V$ denote the natural module (over $F$ ).

First suppose that $d$ is even. Then $\phi(x)=\phi_{1}(x) \phi_{2}(x)$ where $\phi_{i}(x)$ has degree $d / 2$ and $\phi_{2}(x)$ is the Galois conjugate of $\phi_{1}(x)$ under the $q$ power map.

It follows that $V=V_{1} \oplus V_{2}$ where $V_{i}$ is the kernel of $\phi_{i}(A)^{n}$. Then $V_{1}$ and $V_{2}$ are totally singular spaces (with respect to the Hermitian form) and $A$ has characteristic polynomial a power of $\phi_{i}(x)$ on $V_{i}$.

Let $A_{i}$ denote the restriction of $A$ to $V_{i}$. Clearly the centralizer of $A$ preserves each $V_{i}$. Note that if $g \in \mathrm{GU}(n, q)$ fixes each $V_{i}$, then with respect to an appropriate basis if $g_{i}$ denotes $g$ restricted to $V_{i}$, then $g_{2}=\bar{g}_{1}^{-\top}$. The subgroup stabilizing $V_{1}$ and $V_{2}$ is isomorphic to $\operatorname{GL}\left(n / 2, q^{2}\right)$ and the centralizer of $A$ in $U$ is isomorphic to the centralizer of $A_{1}$ in $\operatorname{GL}\left(n, q^{2}\right)$. The result then follows by the result for GL.

Now suppose that $d$ is odd. Then $\phi(x)$ remains irreducible over $F$. By the uniqueness of the canonical forms, we may assume that $V$ is an orthogonal direct sum of subspaces $V_{i}$ such that on $V_{i}$ the rational canonical form has all blocks the same (i.e. the minimal and characteristic polynomials are both powers of $\phi(x))$. 
By considering the GL case (over $k$ ) we see that the reductive part of the centralizer (up to conjugacy) preserves each $V_{i}$. Thus, it suffices to assume that $V=V_{i}$. In the notation given above, this means that $\lambda_{1}=\lambda, e_{1}=e$ and $d e \lambda=n$.

First suppose that $\phi(x)$ is the minimal polynomial of $A$ (i.e. $A$ is a semisimple element and $\lambda=1)$. Then we can decompose the space as a direct sum of $e$ orthogonal spaces each of dimension $d$ so that $A$ acts irreducibly on each space. By the GL case, the centralizer in $\operatorname{GL}(n, F)$ is just $\operatorname{GL}\left(e, q^{2 d}\right)$. We can view the standard Hermitian form as inducing one on the $e$ dimensional vector space over $\mathbb{F}_{q^{2 d}}$ and clearly $\operatorname{GL}\left(e, q^{2 d}\right) \cap \mathrm{GU}(d e, q)=$ $\mathrm{GU}(e, q)$ as claimed.

Finally suppose that $\lambda>1$. Then we can decompose the space as $V_{1} \oplus$ $\ldots \oplus V_{\lambda}$ (this is not an orthogonal decomposition and indeed $V_{1}$ will be totally singular) so that the matrix of $A$ with respect to this decomposition is

$$
\left(\begin{array}{ccccc}
B & I & 0 & \ldots & 0 \\
0 & B & I & \ldots & 0 \\
& & \ldots & & \\
0 & 0 & 0 & \ldots & B
\end{array}\right)
$$

Here $B$ is semisimple and has minimal polynomial $\phi(x)$ and characteristic polynomial $\phi(x)^{e}$. We can take the Hermitian form preserved by this matrix to be in block diagonal form (as in the above equation) with the antidiagonal blocks all being $I$ and all other blocks being 0 . So we need to compute the centralizer with respect to the unitary group preserving this form and as before we only need to compute the reductive part of this centralizer. Again, considering the case of GL, we see that the reductive part of the centralizer will be block diagonal. It is straightforward to see that if $x$ is block diagonal centralizing $A$, then all diagonal blocks are the same. By the previous case this implies that $x \in \mathrm{GU}\left(e, q^{d}\right)$ and clearly any such element commutes with $A$, whence the result.

Lemma 3.5 is a unitary analog of Lemma 2.3 and the proof is identical given the previous results.

Lemma 3.5. The orbits of $\mathrm{GU}(n, q)$ on its Lie algebra correspond to $\left\{\lambda_{\phi}\right\}$ satisfying

$$
\sum_{\phi} d(\phi)\left|\lambda_{\phi}\right|=n
$$

that is to the possible rational canonical forms of an element of $\operatorname{Mat}(n, q)$. The size of the orbit corresponding to data $\left\{\lambda_{\phi}\right\}$ is

$$
\frac{|\mathrm{GU}(n, q)|}{\prod_{\phi} C_{\phi}(\lambda)}
$$


Here

$$
C_{\phi}(\lambda)=q^{d(\phi) \sum_{i}\left(\lambda_{\phi, i}^{\prime}\right)^{2}} \prod_{i}\left(1 / q^{d(\phi)}\right)_{m_{i}\left(\lambda_{\phi}\right)}
$$

if $d(\phi)$ is even, and

$$
C_{\phi}(\lambda)=q^{d(\phi) \sum_{i}\left(\lambda_{\phi, i}^{\prime}\right)^{2}} \prod_{i}\left(1 / q^{d(\phi)}\right)_{m_{i}\left(\lambda_{\phi}\right), q \mapsto-q}
$$

if $d(\phi)$ is odd. Here the notation $\left(1 / q^{d(\phi)}\right)_{m_{i}\left(\lambda_{\phi}\right), q \mapsto-q}$ means that we replace $q$ by $-q$ in the expression $\left(1 / q^{d(\phi)}\right)_{m_{i}\left(\lambda_{\phi}\right)}$.

Lemma 3.6 is a unitary analog of Lemma 2.4, and the proof is identical given the previous results.

Lemma 3.6. Let $x$ be an element of the Lie algebra of $\mathrm{GU}(n, q)$, with data $\left\{\lambda_{\phi}\right\}$. Then the number of elements of the Lie algebra of $\operatorname{GU}(n, q)$ which commute with $x$ is equal to

$$
\prod_{\phi} q^{d(\phi) \sum_{i}\left(\lambda_{\phi, i}^{\prime}\right)^{2}}
$$

Now we prove the unitary analog of the Feit-Fine theorem. Let $U_{0}=1$ and for $n \geq 1$, let $U_{n}$ be the number of ordered pairs of commuting elements of the Lie algebra of $\mathrm{GU}(n, q)$.

\section{Theorem 3.7.}

$$
\sum_{n \geq 0} \frac{U_{n} u^{n}}{|\mathrm{GU}(n, q)|}=\prod_{i \geq 1} \prod_{l \geq 0} \frac{1}{1-(-1)^{l} u^{i} / q^{l-1}}
$$

Proof. Let $\left[u^{n}\right] f(u)$ denote the coefficient of $u^{n}$ in an expression $f(u)$. It follows from Lemmas 3.5 and 3.6 that $U_{n}$ is equal to $|\mathrm{GU}(n, q)|$ multiplied by the coefficient of $u^{n}$ in

$$
\prod_{\substack{\phi \\ d(\phi) \text { even }}} \sum_{\lambda} \frac{u^{|\lambda| d(\phi)}}{\prod_{i}\left(1 / q^{d(\phi)}\right)_{m_{i}(\lambda)}} \prod_{\substack{\phi \\ d(\phi)}} \sum_{\lambda} \frac{u^{|\lambda| d(\phi)}}{\prod_{i}\left(1 / q^{d(\phi)}\right)_{m_{i}(\lambda), q \mapsto-q}} .
$$

This is equal to $|\mathrm{GU}(n, q)|$ multiplied by the coefficient of $u^{n}$ in

$$
\prod_{\substack{\phi \\ d(\phi) \text { even }}} \prod_{i \geq 1} \sum_{m \geq 0} \frac{u^{i m \cdot d(\phi)}}{\left(1 / q^{d(\phi)}\right)_{m}} \prod_{\substack{\phi \\ d(\phi) \text { odd }}} \prod_{i} \sum_{m \geq 0} \frac{u^{i m \cdot d(\phi)}}{\prod_{i}\left(1 / q^{d(\phi)}\right)_{m, q \mapsto-q}} .
$$

By Lemma 2.1, this is equal to

$$
|\mathrm{GU}(n, q)|\left[u^{n}\right] \prod_{\phi} \prod_{i \geq 1} \prod_{l \geq 0} \frac{1}{1-\left(u^{i}(-1)^{l} / q^{l}\right)^{d(\phi)}} .
$$


Switching the order of the products and applying Lemma 2.2 shows that this is equal to

$$
\begin{aligned}
& |\mathrm{GU}(n, q)|\left[u^{n}\right] \prod_{i \geq 1} \prod_{l \geq 0} \prod_{\phi} \frac{1}{1-\left(u^{i}(-1)^{l} / q^{l}\right)^{d(\phi)}} \\
= & |\mathrm{GU}(n, q)|\left[u^{n}\right] \prod_{i \geq 1} \prod_{l \geq 0} \frac{1}{1-(-1)^{l} u^{i} / q^{l-1}} .
\end{aligned}
$$

This completes the proof.

Theorem 3.8 determines the asymptotics of the sequence $U_{n}$.

Theorem 3.8. For q fixed,

$$
\lim _{n \rightarrow \infty} \frac{U_{n}}{q^{n^{2}+n}}=\prod_{i \text { odd }}\left(1-1 / q^{i}\right)^{-1} \prod_{i \text { even }}\left(1-1 / q^{i}\right)^{-\lfloor i / 4\rfloor} .
$$

Proof. We know that

$$
\begin{aligned}
U_{n} & =|\mathrm{GU}(n, q)|\left[u^{n}\right] \prod_{i \geq 1} \prod_{l \geq 0} \frac{1}{1-(-1)^{l} u^{i} / q^{l-1}} \\
& =|\mathrm{GU}(n, q)| q^{n}\left[u^{n}\right] \prod_{i \geq 1} \prod_{l \geq 0} \frac{1}{1-(-1)^{l} u^{i} / q^{i+l-1}} .
\end{aligned}
$$

Thus

$$
\lim _{n \rightarrow \infty} \frac{U_{n}}{q^{n^{2}+n}}=\prod_{j \geq 1}\left(1-(-1)^{j} / q^{j}\right) \lim _{n \rightarrow \infty}\left[u^{n}\right] \prod_{i \geq 1} \prod_{l \geq 0} \frac{1}{1-(-1)^{l} u^{i} / q^{i+l-1}} .
$$

Since the $i=1, l=0$ case of $1 /\left(1-(-1)^{l} u^{i} / q^{i+l-1}\right)$ is equal to $1 /(1-u)$, it follows from Lemma 2.5 that

$$
\begin{aligned}
\lim _{n \rightarrow \infty} \frac{U_{n}}{q^{n^{2}+n}}= & \frac{1}{(1-1 / q)} \cdot \frac{1}{\left(1+1 / q^{2}\right)\left(1-1 / q^{2}\right)} \\
& \cdot \frac{1}{\left(1-1 / q^{3}\right)\left(1+1 / q^{3}\right)\left(1-1 / q^{3}\right)} \\
& \cdot \frac{1}{\left(1+1 / q^{4}\right)\left(1-1 / q^{4}\right)\left(1+1 / q^{4}\right)\left(1-1 / q^{4}\right)} \cdots \\
= & \prod_{i \text { odd }\left(1-1 / q^{i}\right)^{-1} \cdot \frac{1}{\left(1+1 / q^{2}\right)\left(1-1 / q^{2}\right)}} \cdot \frac{1}{\left(1+1 / q^{3}\right)\left(1-1 / q^{3}\right)} \\
& \cdot \frac{1}{\left(1+1 / q^{4}\right)\left(1-1 / q^{4}\right)\left(1+1 / q^{4}\right)\left(1-1 / q^{4}\right)} \cdots
\end{aligned}
$$

Writing $\left(1+1 / q^{k}\right)\left(1-1 / q^{k}\right)=\left(1-1 / q^{2 k}\right)$ shows that this is equal to

$$
\prod_{i \text { odd }}\left(1-1 / q^{i}\right)^{-1} \prod_{i \text { even }}\left(1-1 / q^{i}\right)^{-\lfloor i / 4\rfloor}
$$


Remark: Let $\operatorname{Com}(G)$ denote the number of ordered pairs of commuting elements of a finite group $G$. As mentioned earlier, $\operatorname{Com}(G)$ is equal to $k(G)|G|$, where $k(G)$ is the number of conjugacy classes of $G$. From [FG],

$$
\lim _{n \rightarrow \infty} \frac{k(\mathrm{GU}(n, q))}{q^{n}}=\prod_{i \geq 1} \frac{\left(1+1 / q^{i}\right)}{\left(1-1 / q^{i}\right)} .
$$

Thus,

$$
\begin{aligned}
\lim _{n \rightarrow \infty} \frac{\operatorname{Com}(\mathrm{GU}(n, q))}{q^{n^{2}+n}} & =\prod_{i \geq 1} \frac{\left(1+1 / q^{i}\right)}{\left(1-1 / q^{i}\right)} \lim _{n \rightarrow \infty} \frac{|\mathrm{GU}(n, q)|}{q^{n^{2}}} \\
& =\prod_{i \text { odd }} \frac{\left(1+1 / q^{i}\right)}{\left(1-1 / q^{i}\right)} \prod_{i \geq 1}\left(1+1 / q^{i}\right) .
\end{aligned}
$$

Next we enumerate pairs of commuting nilpotent matrices in the Lie algebra of $\mathrm{GU}(n, q)$. Let $N U_{0}=1$, and for $n \geq 1$, let $N U_{n}$ be the number of ordered pairs of commuting $n \times n$ nilpotent matrices in the Lie algebra of $\mathrm{GU}(n, q)$.

\section{Theorem 3.9.}

$$
\sum_{n \geq 0} \frac{N U_{n} u^{n}}{|\mathrm{GU}(n, q)|}=\prod_{i \geq 1} \prod_{l \geq 1} \frac{1}{\left(1+(-1)^{l} u^{i} / q^{l}\right)} .
$$

We next state a lemma about counting nilpotent elements in a Lie subalgebra.

Lemma 3.10. Let $G$ be a connected algebraic group with Lie algebra $\mathfrak{g}$ over an algebraically closed field of characteristic $p$. Let $F$ be a Lang-Steinberg endomorphism of $G$ (so $G^{F}$ is a finite group). The number of nilpotent elements in $(\operatorname{Lie}(G))^{F}$ is $q^{\operatorname{dim} G-\text { rank } G}$ (where $q=q_{F}$ is the size of the field associated with $F$ ).

Proof. Let $Q$ be the unipotent radical of $G$. Of course $Q$ is $F$-invariant. Let $\mathfrak{h}$ be the Lie algebra of $Q$. Then $\mathfrak{h}$ consists of nilpotent elements and $\mathfrak{h}^{F}$ will have cardinality $q^{\operatorname{dim} Q}$. Thus, we can pass to the case that $G$ is reductive. In that case, we just apply a result of Lehrer [Le, Cor. 1.15] (this also follows by a result of Steinberg [St in good characteristic - Steinberg proves the corresponding result for unipotent elements).

Lemma 3.11. The number of nilpotent $n \times n$ matrices which are in the Lie algebra of $\mathrm{GU}(n, q)$ and which commute with a fixed nilpotent matrix of type $\lambda$ in the Lie algebra of $\mathrm{GU}(n, q)$ is equal to

$$
q^{\sum_{i}\left(\lambda_{i}^{\prime}\right)^{2}-\sum_{i} m_{i}(\lambda)}
$$

Proof. Let $A \in \mathfrak{h}$ be nilpotent of type $\lambda$. As we have already noted, the dimension of the centralizer of $A$ is $\sum_{i}\left(\lambda_{i}^{\prime}\right)^{2}$. We have also seen that the 
reductive part of the centralizer of $A$ is a direct product of $\operatorname{GL}\left(m_{i}\left(\lambda_{i}\right)\right)$, whence the rank of the centralizer is $\sum_{i} m_{i}(\lambda)$. Thus, the result follows by Lemma 3.10 .

Now we prove Theorem 3.9 .

Proof. (Of Theorem 3.9) From Lemma 3.5 we know that the total number of elements of the Lie algebra of $\mathrm{GU}(n, q)$ which are nilpotent of type $\lambda$ is equal to

$$
\frac{|\mathrm{GU}(n, q)|}{q^{\sum\left(\lambda_{i}^{\prime}\right)^{2}} \prod_{i}(1 / q)_{m_{i}(\lambda), q \mapsto-q}} .
$$

Here the notation $(1 / q)_{m_{i}(\lambda), q \mapsto-q}$ means that we replace $q$ by $-q$ in the expression $(1 / q)_{m_{i}(\lambda)}$.

Combining this with Lemma 3.11 gives that

$$
\begin{aligned}
N U_{n} & =|\mathrm{GU}(n, q)| \sum_{|\lambda|=n} \frac{1}{\prod_{i} q^{m_{i}(\lambda)}(1 / q)_{m_{i}(\lambda), q \mapsto-q}} \\
& =|\mathrm{GU}(n, q)|\left[u^{n}\right] \prod_{i \geq 1} \sum_{m \geq 0} \frac{u^{i m}}{q^{m}(1 / q)_{m, q \mapsto-q}} \\
& =|\mathrm{GU}(n, q)|\left[u^{n}\right] \prod_{i \geq 1} \sum_{m \geq 0} \frac{\left[(-1) u^{i}\right]^{m}}{\left[q^{m}(1 / q)_{m}\right]_{q \mapsto-q}} .
\end{aligned}
$$

It follows from Lemma 2.1 that

$$
\sum_{m \geq 0} \frac{\left[(-1) u^{i}\right]^{m}}{q^{m}(1 / q)_{m}}=\prod_{l \geq 1} \frac{1}{1+u^{i} / q^{l}} .
$$

Replacing $q$ by $-q$ gives that

$$
\sum_{m \geq 0} \frac{\left[(-1) u^{i}\right]^{m}}{\left[q^{m}(1 / q)_{m}\right]_{q \mapsto-q}}=\prod_{l \geq 1} \frac{1}{1+(-1)^{l} u^{i} / q^{l}} .
$$

Hence

$$
N U_{n}=|\mathrm{GU}(n, q)|\left[u^{n}\right] \prod_{i \geq 1} \prod_{l \geq 1} \frac{1}{\left(1+(-1)^{l} u^{i} / q^{l}\right)} .
$$

\section{Symplectic Groups}

Here we consider the symplectic case. There are several extra difficulties in characteristic 2. So let $F=\mathbb{F}_{q}$ with $q$ an odd prime power. Let $G=$ $\operatorname{Sp}(2 n, q)$. Let $\mathfrak{s p}$ be the Lie algebra of $G$. This can be viewed in a number of ways.

One way is as follows. Let $J$ denote the skew symmetric matrix defining $G$ (i.e. $G$ is the stabilizer of $J$ ). Then

$$
\mathfrak{s p}:=\left\{A \in \operatorname{Mat}(n, q) \mid A J=(A J)^{\top}\right\} .
$$


We can also view $\mathfrak{s p}$ as the linear transformations on the natural module $V$ for $G$ which are self adjoint with respect to the alternating form defining $G$. Note that $V$ is a self-dual $G$-module and so $V \cong V^{*}$ (where $V^{*}$ denotes the dual module).

First let us make some remarks if we work over the algebraic closure $k$ of $F$. Note that if $x \in \mathfrak{s p}$, then if $0 \neq \alpha$ is an eigenvalue of $x$, so is $-\alpha$ and the generalized $\alpha$ eigenspace of $x$ is totally singular. The sum of the generalized $\alpha$ and $-\alpha$ eigenspaces is nonsingular and the Jordan form of $x$ on the $\alpha$ space is the same as the $-\alpha$ space. Moreover the centralizer in $\operatorname{Sp}(2 m)$ is just the centralizer of $x$ in $\operatorname{GL}(m)$ (on the $\alpha$ ) space. In particular, the centralizer is connected.

We first compute the dimension of the centralizer of an element $x \in \mathfrak{s p}$. As usual, we reduce to the case where the characteristic polynomial of $x$ is a power of an irreducible polynomial $\phi(x)$ or the product of two irreducible polynomials $\phi_{i}(x)$ where $\phi_{2}(x)=(-1)^{d} \phi_{1}(-x)$ where $d$ is the degree of $\phi_{i}$. In the first case note that either $\phi(x)=x$ or $\phi(x)$ has even degree $2 d$ (and $\phi(x)=\phi(-x)$.

First consider the case that $\phi(x)=x$ (i.e. $x$ is nilpotent). Then the orbit of $x$ under $\operatorname{Sp}(2 n, k)$ is determined by the Jordan form of $x$ which can be described as a partition of $n$. Let $\lambda_{1} \geq \lambda_{2} \geq \ldots \geq \lambda_{r}$ (such a partition corresponds to an element of $\mathfrak{s p}$ if and only if the multiplicity of the odd pieces in the partition is even).

Recall that $\mathfrak{s p}$ as a module either for $G$ or for the Lie algebra is isomorphic to $\operatorname{Sym}^{2}(V)$ and this is a direct summand of $V \otimes V$ (which is isomorphic to $V \otimes V^{*}$ as a module for $x$ ) since we are assuming that $q$ is odd. Since the dimension of the fixed point space on $V \otimes V$ depends only on the partition and not on the characteristic, the same is true for the dimension of the fixed point space on $\operatorname{Sym}^{2}(V)$. This is well known (for example, this can be computed in characteristic 0 using the decomposition of $\operatorname{Sym}^{2}(V)$ as an $\mathrm{SL}(2)$-module). This proves the first expression in the following lemma.

Lemma 4.1. Let $x \in \mathfrak{s p}$ be nilpotent with Jordan block sizes $\lambda_{1} \geq \lambda_{2} \geq$ $\ldots \geq \lambda_{r}$ on the natural module. Then the dimension of the centralizer of $x$ in $\mathfrak{s p}$ is equal to both:

(1)

$$
\sum_{j<i} \lambda_{i}+\sum_{i}\left\lceil\lambda_{i} / 2\right\rceil
$$

and

$$
\sum_{i}\left(\lambda_{i}^{\prime}\right)^{2} / 2+o(\lambda) / 2
$$

where $o(\lambda)$ is the number of odd parts of $\lambda$. 
Proof. The expression in part 1 of the lemma is equal to

$$
\begin{aligned}
\sum_{j<i} \lambda_{i}+\sum_{i} \lambda_{i} / 2+o(\lambda) / 2 & =\sum_{i} \lambda_{i}(i-1)+|\lambda| / 2+o(\lambda) / 2 \\
& =\sum_{i}\left(\begin{array}{c}
\lambda_{i}^{\prime} \\
2
\end{array}\right)+|\lambda| / 2+o(\lambda) / 2 \\
& =\sum_{i}\left(\lambda_{i}^{\prime}\right)^{2} / 2+o(\lambda) / 2 .
\end{aligned}
$$

Now consider the case that $\phi(x) \neq x$.

Then the class of the element is determined by $\phi(x)$ (or the $\phi_{i}(x)$ ) and a partition of $n / d$.

To compute the dimension of the centralizer of $x$ in $\mathfrak{s p}$, we can work over the algebraic closure (or any extension field) and so reduce to the case where the characteristic polynomial is a power of $\phi_{1}(x) \phi_{2}(x)$. Then we can write $V=V_{1} \oplus V_{2}$ where each $V_{i}$ is a maximal totally singular subspace, $V_{i}$ is invariant under $x$ and the characteristic polynomial of $x$ on $V_{i}$ is a power of $\phi_{i}(x)$. Then we see that the centralizer of $x$ in $\mathfrak{s p}$ is isomorphic to the centralizer of $x_{i}$ (the restriction of $x$ to $V_{i}$ ) in the algebra of linear transformations of $V_{i}$ and so the result for GL gives Theorem 4.2 below.

Recall that Jordan canonical form associates to each monic, irreducible polynomial $\phi$ over $\mathbb{F}_{q}$ a partition $\lambda_{\phi}$ such that

$$
\sum_{\phi} d(\phi)\left|\lambda_{\phi}\right|=2 n
$$

This data arises from an element of $\mathfrak{s p}$ if and only if:

(1) The partition $\lambda_{x}$ has all odd parts occur with even multiplicity

(2) For every $\phi$, the partition $\lambda_{\phi}$ is equal to the partition $\lambda_{\bar{\phi}}$, where $\bar{\phi}$ is defined as $(-1)^{d(\phi)} \phi(-x)$.

Thus, we have proved:

Theorem 4.2. If $\left\{\lambda_{\phi}\right\}$ is the data corresponding to an element of $\mathfrak{s p}$, then the dimension of its centralizer in $\mathfrak{s p}$ is equal to

$$
\sum_{i}\left(\lambda_{x, i}^{\prime}\right)^{2} / 2+o\left(\lambda_{x}\right) / 2+\sum_{\substack{\phi=\bar{\phi} \\ \phi \neq x}} d(\phi) \sum_{i}\left(\lambda_{\phi, i}^{\prime}\right)^{2} / 2+\sum_{\substack{\{\phi, \bar{\phi}\} \\ \phi \neq \bar{\phi}}} d(\phi) \sum_{i}\left(\lambda_{\phi, i}^{\prime}\right)^{2} .
$$

Finally, we need to count how many elements there are in $\mathfrak{s p}$ with a given canonical form. This amounts to computing the centralizers in Sp. As in the discussion for centralizers in $\mathfrak{s p}$, we can reduce to the three cases as above. It is a bit more involved as we now need the order of the centralizer rather than just the dimension.

For nilpotent elements, the centralizer is not usually connected in the algebraic group and so the orbits break up over the finite field. However, 
since the centralizer dimension in the Lie algebra depends only the orbit over $k$, we only need to know the following.

Lemma 4.3. The number of nilpotent elements in $\mathfrak{s p}$ corresponding to a partition $\lambda_{1} \geq \lambda_{2} \geq \ldots \geq \lambda_{r}$ is

$$
\frac{|\operatorname{Sp}(2 n, q)|}{q^{\sum_{i}\left(\lambda_{i}^{\prime}\right)^{2} / 2+o(\lambda) / 2} \prod_{i}\left(1-1 / q^{2}\right) \cdots\left(1-q^{2\left\lfloor m_{i}(\lambda) / 2\right\rfloor}\right)}
$$

Proof. Since we are in good characteristic, the number of nilpotent elements with a given Jordan form is the same as the number of unipotent elements in $\operatorname{Sp}(2 n, q)$ with the same distribution of Jordan blocks. This number is determined in $[\mathrm{F}]$.

For the elements in $\mathfrak{s p}$ that do not have 0 as an eigenvalue, we have seen that the centralizers in the algebraic group are connected, whence the $\operatorname{Sp}(2 n, k)$-orbits intersect $\mathfrak{s p}(2 n, q)$ are just the $\operatorname{Sp}(2 n, q)$ orbits. So we just need to compute the centralizers in $\operatorname{Sp}(2 n, q)$.

The next result gives the number of elements in $\mathfrak{s p}(2 n, q)$ with a given Jordan canonical form.

Theorem 4.4. If $\left\{\lambda_{\phi}\right\}$ is the data corresponding to an element $x$ of $\mathfrak{s p}$, then the number of elements of $\mathfrak{s p}$ corresponding to this data is equal to $|\mathrm{Sp}(2 n, q)|$ multiplied by:

$$
\begin{aligned}
& \frac{1}{q^{\sum_{i}\left(\lambda_{x, i}^{\prime}\right)^{2} / 2+o\left(\lambda_{x}\right) / 2} \prod_{i}\left(1-1 / q^{2}\right) \cdots\left(1-1 / q^{2\left\lfloor m_{i}\left(\lambda_{x}\right) / 2\right\rfloor}\right)} \\
& \cdot \prod_{\substack{\phi=\bar{\phi} \\
\phi \neq x}} \frac{1}{q^{d(\phi) \sum_{i}\left(\lambda_{\phi, i}^{\prime}\right)^{2} / 2} \prod_{i}\left(1+1 / q^{d(\phi) / 2}\right) \cdots\left(1-(-1)^{m_{i}\left(\lambda_{\phi}\right)} / q^{m_{i}\left(\lambda_{\phi}\right) d(\phi) / 2}\right)} \\
& \cdot \prod_{\substack{\{\phi, \bar{\phi}\} \\
\phi \neq \bar{\phi}}} \frac{1}{q^{d(\phi) \sum_{i}\left(\lambda_{\phi, i}^{\prime}\right)^{2}} \prod_{i}\left(1-1 / q^{d(\phi)}\right) \cdots\left(1-1 / q^{m_{i}\left(\lambda_{\phi}\right) d(\phi)}\right)}
\end{aligned}
$$

Proof. By the discussion before Lemma 4.1 and Lemma 4.3 it suffices to reduce to the case where $\phi \neq x$ and $\phi$ is either irreducible (and $\phi(x)=$ $\phi(-x))$ or $\phi=\phi_{1} \phi_{2}$ where $\phi_{1}$ is irreducible and $\phi_{2}$ is the dual polynomial of $\phi_{1}$ (i.e. its roots are the negatives of the roots of $\phi_{1}$ ).

In the latter case, $V$ decomposes as a direct sum of two totally isotropic spaces $V_{1} \oplus V_{2}$ where the element has characteristic polynomial a power of $\phi_{i}$ on $V_{i}$ and the action on $V_{2}$ is the dual of the action on $V_{1}$. Thus, the centralizer of $x$ is isomorphic to the centralizer in $\mathrm{GL}\left(V_{1}\right)$ of $x$ restricted to $V_{1}$ and the result follows by Lemma 2.4.

So it remains to consider the case that $\phi$ is irreducible. The unipotent radical of the centralizer has order $q^{\operatorname{dim} Q}$ where $Q$ is the unipotent radical of the centralizer in the algebraic group (and so can be computed as above). So we only have to identify the reductive part. Modulo the unipotent radical, we see that we can reduce to the case where all parts of the partition have 
the same size $e$ (this can be seen in the algebraic group). Let $m$ denote the this multiplicity and let $2 d$ be the degree of $\phi$.

If we pass to a quadratic extension of our finite field, we are in the previous case and so the reductive part of the centralizer is $\operatorname{GL}\left(m, q^{2 d}\right)$. Arguing as we did in the unitary case, we see that the reductive part of the centralizer is $\mathrm{GU}\left(m, q^{d}\right)$.

Recall that $\bar{\phi}$ is defined as $(-1)^{d(\phi)} \phi(-x)$. Note that if a monic irreducible polynomial $\phi \neq x$ satisfies $\bar{\phi}=\phi$, then $\phi$ has even degree. We let $\bar{N}(2 d, q)$ denote the number of monic irreducible polynomials $\phi$ of degree $2 d$ such that $\bar{\phi}=\phi$. It is also helpful to let $\bar{M}(d, q)$ denote the number of unordered pairs $\{\phi, \bar{\phi}\}$ of monic, irreducible, degree $d$ polynomials such that $\phi \neq \bar{\phi}$.

The following enumerative lemma will be helpful. It is a symplectic analog of Lemma 2.2.

Lemma 4.5.

$$
\begin{gathered}
\prod_{d \geq 1}\left(1-u^{d}\right)^{-\bar{N}(2 d, q)} \prod_{d \geq 1}\left(1-u^{d}\right)^{-\bar{M}(d, q)}=\frac{1-u}{1-q u} \\
\prod_{d \geq 1}\left(1+u^{d}\right)^{-\bar{N}(2 d, q)} \prod_{d \geq 1}\left(1-u^{d}\right)^{-\bar{M}(d, q)}=1
\end{gathered}
$$

Proof. To prove the first assertion, note that any monic polynomial (not necessarily irreducible) which is invariant under the involution $f(x) \mapsto$ $(-1)^{d(f)} f(-x)$ factors uniquely into irreducibles as a product of powers of $\phi$ where $\bar{\phi}=\phi$ and of powers of $\phi \bar{\phi}$ where $\phi \neq \bar{\phi}$. Hence the coefficient of $u^{n}$ in

$$
(1-u)^{-1} \prod_{d \geq 1}\left(1-u^{d}\right)^{-\bar{N}(2 d, q)} \prod_{d \geq 1}\left(1-u^{d}\right)^{-\bar{M}(d, q)}
$$

is equal to the number of monic degree $2 n$ polynomials $f$ such that $f(x)=$ $f(-x)$. This is equal to $q^{n}$, which is the coefficient of $u^{n}$ in $\frac{1}{1-q u}$, proving the first part of the theorem.

To prove the second part of the theorem, we claim that

$$
(1-u)^{-1} \prod_{d \geq 1}\left(1-u^{2 d}\right)^{-\bar{N}(2 d, q)} \prod_{d \geq 1}\left(1-u^{d}\right)^{-2 \bar{M}(d, q)}=\frac{1}{1-q u} .
$$

Indeed, the claimed equation follows since the coefficient of $u^{n}$ in the left hand side counts the total number of monic degree $n$ polynomials, as does the coefficient of $u^{n}$ in $1 /(1-q u)$. Dividing the claimed equation by the first assertion of the theorem immediately proves the second assertion of the theorem.

Let $S_{0}=1$, and for $n \geq 1$, let $S_{n}$ be the number of ordered pairs of commuting elements of the Lie algebra of $\operatorname{Sp}(2 n, q)$. Armed with the above results, we can now derive a generating function for the sequence $S_{n}$. 


\section{Theorem 4.6.}

$$
\sum_{n \geq 0} \frac{S_{n} u^{n}}{|\operatorname{Sp}(2 n, q)|}=\frac{\prod_{i \geq 1}\left(1+u^{i}\right)}{\prod_{i \geq 1} \prod_{l \geq 0}\left(1-u^{i} / q^{2 l-1}\right)} .
$$

Proof. It is immediate from Theorems 4.2 and 4.4 that $S_{n}$ is equal to $|\operatorname{Sp}(2 n, q)|$ multiplied by the coefficient of $u^{2 n}$ in $A B C$ where

$$
\begin{gathered}
A=\sum_{\substack{\lambda \\
i \text { odd } \Longrightarrow m_{i}(\lambda) \text { even }}} \frac{u^{|\lambda|}}{\prod_{i}\left(1-1 / q^{2}\right)\left(1-1 / q^{4}\right) \cdots\left(1-1 / q^{2\left\lfloor m_{i}(\lambda) / 2\right\rfloor}\right)} \\
B=\prod_{\substack{\phi=\bar{\phi} \\
\phi \neq x}} \sum_{\lambda} \frac{u^{d(\phi)|\lambda|}}{\prod_{i}\left(1+1 / q^{d(\phi) / 2}\right) \cdots\left(1-(-1)^{\left.m_{i}(\lambda) / q^{m_{i}(\lambda) d(\phi) / 2}\right)}\right.} \\
C=\prod_{\substack{\{\phi, \bar{\phi}\} \\
\phi \neq \bar{\phi}}} \sum_{\lambda} \frac{u^{2 d(\phi)|\lambda|}}{\prod_{i}\left(1-1 / q^{d(\phi)}\right) \cdots\left(1-1 / q^{m_{i}(\lambda) d(\phi)}\right)} .
\end{gathered}
$$

Note that in $A$, the sum is over all partitions such that the odd parts occur with even multiplicity, and that in $B$ and $C$, the sum is over all partitions.

Note that $A=(A 1)(A 2)$ where

$$
A 1=\prod_{i \text { odd }} \sum_{m \text { even }} \frac{u^{i m}}{\left(1-1 / q^{2}\right)\left(1-1 / q^{4}\right) \cdots\left(1-1 / q^{m}\right)}
$$

and $A 2$ is equal to

$$
\prod_{\substack{i \text { even } \\ i \geq 2}}\left[\sum_{m \text { even }} \frac{u^{i m}}{\left(1-1 / q^{2}\right) \cdots\left(1-1 / q^{m}\right)}+\sum_{m \text { odd }} \frac{u^{i m}}{\left(1-1 / q^{2}\right) \cdots\left(1-1 / q^{m-1}\right)}\right]
$$

Applying Lemma 2.1 gives that

$$
\begin{aligned}
& A 1=\prod_{i \text { odd } l \geq 0} \prod_{l} \frac{1}{\left(1-u^{2 i} / q^{2 l}\right)} \\
& A 2=\prod_{\substack{i \text { even } \\
i \geq 2}}\left(1+u^{i}\right) \prod_{l \geq 0} \frac{1}{\left(1-u^{2 i} / q^{2 l}\right)} .
\end{aligned}
$$

Next observe that

$$
\begin{aligned}
B & =\prod_{\substack{\phi=\bar{\phi} \\
\phi \neq x}} \prod_{i \geq 1} \sum_{m \geq 0} \frac{u^{i m d(\phi)}}{\left(1+1 / q^{d(\phi) / 2}\right) \cdots\left(1-(-1)^{m} / q^{m d(\phi) / 2}\right)} \\
& =\prod_{\substack{\phi=\bar{\phi} \\
\phi \neq x}} \prod_{i \geq 1} \prod_{l \geq 0} \frac{1}{\left(1-(-1)^{l} u^{i d(\phi)} / q^{l d(\phi) / 2}\right)}
\end{aligned}
$$

where the second equality used Lemma 2.1. 
Similarly,

$$
\begin{aligned}
C & =\prod_{\substack{\{\phi, \bar{\phi}\} \\
\phi \neq \bar{\phi}}} \prod_{i \geq 1} \sum_{m \geq 0} \frac{u^{2 i m d(\phi)}}{\left(1-1 / q^{d(\phi)}\right) \cdots\left(1-1 / q^{m d(\phi)}\right)} \\
& =\prod_{\substack{\{\phi, \bar{\phi}\} \\
\phi \neq \bar{\phi}}} \prod_{i \geq 1} \prod_{l \geq 0} \frac{1}{\left(1-u^{2 i d(\phi)} / q^{l d(\phi)}\right)} .
\end{aligned}
$$

Switching the order of the products in $B$ and $C$, and then applying both parts of Lemma 4.5 gives that

$$
\begin{aligned}
B C & =\prod_{l \geq 0} \prod_{i \geq 1}\left[\prod_{d \geq 1}\left(1-(-1)^{l} u^{2 i d} / q^{l d}\right)^{-\bar{N}(2 d, q)} \prod_{d \geq 1}\left(1-u^{2 i d} / q^{l d}\right)^{-\bar{M}(d, q)}\right] \\
& =\prod_{\substack{l \text { even } \\
l \geq 0}} \prod_{i \geq 1} \frac{\left(1-u^{2 i} / q^{l}\right)}{\left(1-u^{2 i} / q^{l-1}\right)} .
\end{aligned}
$$

Hence

$$
A B C=(A 1)(A 2)(B C)=\frac{\prod_{i \geq 1}\left(1+u^{2 i}\right)}{\prod_{i \geq 1} \prod_{l \geq 0}\left(1-u^{2 i} / q^{2 l-1}\right)},
$$

which proves the theorem.

Next we use Theorem 4.6 to obtain asymptotic information about the number of commuting pairs in the Lie algebra of $\operatorname{Sp}(2 n, q)$.

Theorem 4.7. For q fixed,

$$
\lim _{n \rightarrow \infty} \frac{S_{n}}{q^{2 n^{2}+2 n}}=\prod_{i \geq 1}\left(1+1 / q^{i}\right)\left(1-1 / q^{i}\right)^{-\lfloor(i+1) / 2\rfloor} .
$$

Proof. We know from Theorem 4.6 that

$$
\begin{aligned}
S_{n}= & |\operatorname{Sp}(2 n, q)|\left[u^{n}\right] \frac{\prod_{i \geq 1}\left(1+u^{i}\right)}{\prod_{i \geq 1} \prod_{l \geq 0}\left(1-u^{i} / q^{2 l-1}\right)} \\
= & |\operatorname{Sp}(2 n, q)| q^{n}\left[u^{n}\right] \frac{\prod_{i \geq 1}\left(1+u^{i} / q^{i}\right)}{\prod_{i \geq 1} \prod_{l \geq 0}\left(1-u^{i} / q^{2 l+i-1}\right)} \\
= & q^{2 n^{2}+2 n}\left(1-1 / q^{2}\right) \cdots\left(1-1 / q^{2 n}\right) \\
& \cdot\left[u^{n}\right] \frac{\prod_{i \geq 1}\left(1+u^{i} / q^{i}\right)}{\prod_{i \geq 1} \prod_{l \geq 0}\left(1-u^{i} / q^{2 l+i-1}\right)} .
\end{aligned}
$$

Thus

$$
\lim _{n \rightarrow \infty} \frac{S_{n}}{q^{2 n^{2}+2 n}}=\prod_{j \geq 1}\left(1-1 / q^{2 j}\right) \lim _{n \rightarrow \infty}\left[u^{n}\right] \frac{\prod_{i \geq 1}\left(1+u^{i} / q^{i}\right)}{\prod_{i \geq 1} \prod_{l \geq 0}\left(1-u^{i} / q^{2 l+i-1}\right)} .
$$


Since the $i=1, l=0$ case of $1 /\left(1-u^{i} / q^{2 l+i-1}\right)$ is equal to $1 /(1-u)$, it follows from Lemma 2.5 and elementary manipulations that

$$
\prod_{j \geq 1}\left(1-1 / q^{2 j}\right) \lim _{n \rightarrow \infty}\left[u^{n}\right] \frac{\prod_{i \geq 1}\left(1+u^{i} / q^{i}\right)}{\prod_{i \geq 1} \prod_{l \geq 0}\left(1-u^{i} / q^{2 l+i-1}\right)}
$$

is equal to

$$
\prod_{i \geq 1}\left(1+1 / q^{i}\right)\left(1-1 / q^{i}\right)^{-\lfloor(i+1) / 2\rfloor} .
$$

Remark: Let $\operatorname{Com}(G)$ denote the number of ordered pairs of commuting elements of a finite group $G$. As mentioned earlier, $\operatorname{Com}(G)$ is equal to $k(G)|G|$, where $k(G)$ is the number of conjugacy classes of $G$. From [FG], since $q$ is odd, one has that

$$
\lim _{n \rightarrow \infty} \frac{k(\operatorname{Sp}(2 n, q))}{q^{n}}=\prod_{i \geq 1} \frac{\left(1+1 / q^{i}\right)^{4}}{\left(1-1 / q^{i}\right)} .
$$

Thus,

$$
\begin{aligned}
\lim _{n \rightarrow \infty} \frac{\operatorname{Com}(\operatorname{Sp}(2 n, q))}{q^{2 n^{2}+2 n}} & =\prod_{i \geq 1} \frac{\left(1+1 / q^{i}\right)^{4}}{\left(1-1 / q^{i}\right)} \lim _{n \rightarrow \infty} \frac{|\operatorname{Sp}(2 n, q)|}{q^{2 n^{2}+n}} \\
& =\frac{\prod_{i \geq 1}\left(1+1 / q^{i}\right)^{4}}{\prod_{i \text { odd }}\left(1-1 / q^{i}\right)}
\end{aligned}
$$

Our next goal is to count the number of nilpotent commuting pairs in $\mathfrak{s p}$.

We first require a result of Richardson [R1, Lemma 6.6] (which can be proved quite easily in our special case). Note that for $G=\operatorname{Sp}(2 n, k)$ all characteristics other than 2 are very good.

Lemma 4.8. ([R1]) Let $G$ be a simple algebraic group over an algebraically closed field $k$. Assume that the characteristic is very good for $G$. Let $\mathfrak{g}$ be the Lie algebra of $G$. If $x \in \mathfrak{g}$, then $\{y \in \mathfrak{g} \mid[x, y]=0\}$ is the Lie algebra of $C_{G}(x)$.

Lemma 4.9. If an element $x$ of $\mathfrak{s p}$ is nilpotent of type $\lambda$, then its centralizer in $\mathfrak{s p}$ has size

$$
q^{\sum_{i}\left(\lambda_{i}^{\prime}\right)^{2} / 2+o(\lambda) / 2-\sum_{i}\left\lfloor m_{i}(\lambda) / 2\right\rfloor} .
$$

Proof. Note that we are in very good characteristic. As we have already seen, the dimension of the centralizer in the group of a nilpotent element $x$ is $\sum_{i}\left(\lambda_{i}^{\prime}\right)^{2} / 2+o(\lambda) / 2$. It follows by [LS] that the rank of the centralizer is $\sum_{i}\left\lfloor m_{i}(\lambda) / 2\right\rfloor$. By the result of Richardson above, the same is true for the centralizer of $x$ in $\mathfrak{s p}$. The result now follows by Lemma 3.10,

This leads to the following theorem. In its statement, we let $N S_{0}=1$, and let $N S_{n}$ denote the number of commuting ordered pairs of nilpotent elements in the Lie algebra of $\operatorname{Sp}(2 n, q)$. 
Theorem 4.10.

$$
\sum_{n \geq 0} \frac{N S_{n} u^{n}}{|\operatorname{Sp}(2 n, q)|}=\frac{\prod_{i \geq 1}\left(1+u^{i}\right)}{\prod_{i \geq 1} \prod_{l \geq 0}\left(1-u^{i} / q^{2 l+1}\right)} .
$$

Proof. It follows from Lemmas 4.3 and 4.9 that $N S_{n}$ is equal to

$$
|\operatorname{Sp}(2 n, q)| \sum_{i \text { odd }}^{\substack{|\lambda|=2 n \\ \Rightarrow m_{i}(\lambda) \text { even }}} \frac{1}{\prod_{i}\left(1-1 / q^{2}\right) \cdots\left(1-1 / q^{2\left\lfloor m_{i}(\lambda) / 2\right\rfloor}\right) q^{\left\lfloor m_{i}(\lambda) / 2\right\rfloor}} .
$$

Letting $\left[u^{n}\right] f(u)$ denote the coefficient of $u^{n}$ in a power series $f(u)$, it follows that

$$
N S_{n}=|\operatorname{Sp}(2 n, q)|\left[u^{2 n}\right] A B
$$

where

$$
\begin{aligned}
A & =\prod_{i \text { odd }} \sum_{\text {even }} \frac{u^{i m}}{\left(1-1 / q^{2}\right) \cdots\left(1-1 / q^{m}\right) q^{m / 2}} \\
B & =\prod_{\substack{i \text { even } \\
i \geq 2}} \sum_{m} \frac{u^{i m}}{\left(1-1 / q^{2}\right) \cdots\left(1-1 / q^{2\lfloor m / 2\rfloor}\right) q^{\lfloor m / 2\rfloor}} \\
= & \prod_{\substack{i \text { even } \\
i \geq 2}}\left(1+u^{i}\right) \sum_{m \text { even }} \frac{u^{i m}}{\left(1-1 / q^{2}\right) \cdots\left(1-1 / q^{m}\right) q^{m / 2}} .
\end{aligned}
$$

It follows from Lemma 2.1 that

$$
A B=\frac{\prod_{i \geq 1}\left(1+u^{2 i}\right)}{\prod_{i \geq 1} \prod_{l \geq 0}\left(1-u^{2 i} / q^{2 l+1}\right)},
$$

proving the theorem.

\section{ACKNOWLEDGEMENTS}

Fulman was partially supported by Simons Foundation Grant 400528. Guralnick was partially supported by NSF grant DMS-1600056. The authors thank Matt Young for asking about analogs of the Feit-Fine theorem for other Lie algebras.

\section{REFERENCES}

[A] Andrews, G., The theory of partitions, Cambridge University Press, 1984.

[Ba] Baranovsky, V., The variety of pairs of commuting nilpotent matrices is irreducible, Transform. Groups 6 (2001), 3-8.

[Bs] Basili, R., On the irreducibility of commuting varieties of nilpotent matrices, $J$. Algebra 268 (2003), 58-80.

[BBS] Behrend, K., Bryan, J., and Szendroi, B., Motivic degree zero Donaldson-Thomas invariants, Invent. Math. 192 (2013), 111-160.

[BM] Bryan, J. and Morrison, A., Motivic classes of commuting varieties via power structures, J. Algebraic Geom. 24 (2015), 183-199.

[FF] Feit, W. and Fine, N.J., Pairs of commuting matrices over a finite field, Duke Math. J 27 (1960), 91-94. 
[F] Fulman, J., A probabilistic approach to conjugacy classes in the finite symplectic and orthogonal groups, J. Algebra 234 (2000), 207-224.

[FG] Fulman, J. and Guralnick, R., Bounds on the number and sizes of conjugacy classes in finite Chevalley groups with applications to derangements, Transac. Amer. Math. Soc. 364 (2012), 3023-3070.

[H] Herstein, I.N., Topics in algebra, Second edition, Xerox College Publishing, 1975.

[Lh] Lehrer, G., Rational tori, semisimple orbits and the topology of hyperplane complements, Comment. Math. Helvetici 67 (1992), 226-251.

[Le] Levy, P., Commuting varieties of Lie algebras over fields of prime characteristic, J. Algebra 250 (2002), 473-484.

[LS] Liebeck, M. and Seitz, G., Unipotent and nilpotent classes in simple algebraic groups and Lie algebras, Mathematical Surveys and Monographs, 180, American Mathematical Society, Providence, RI, 2012.

[MT] Motzkin, T.S. and Taussky, O., Pairs of matrices with property L. II, Transac. Amer. Math. Soc. 80 (1955), 387-401.

[Pr] Premet, A., Nilpotent commuting varieties of reductive Lie algebras, Invent. Math. 154 (2003), 653-683.

[R1] Richardson, R.W., Conjugacy classes in Lie algebras and algebraic groups, Ann. of Math. 86 (1967), 1-15.

[R2] Richardson, R.W., Commuting varieties of semisimple Lie algebras and algebraic groups, Compositio Math. 38 (1979), 311-327.

[St] Steinberg, R., Endomorphisms of linear algebraic groups, Memoirs Amer. Math. Society 80 (1968).

[S] Stong, R., Some asymptotic results on finite vector spaces, Adv. in Appl. Math. 9 (1988), 167-199.

[TZ] Taussky, O. and Zassenhaus, H., On the similarity transformation between a matrix and its transpose, Pacific J. Math. 9 (1959), 893-896.

Department of Mathematics, University of Southern California, Los AnGELES, CA, 90089

E-mail address: fulman@usc.edu

Department of Mathematics, University of Southern California, Los AnGELES, CA, 90089

E-mail address: guralnic@usc.edu 